\title{
STOP-Bang questionnaire screening for obstructive sleep apnea among Chinese patients with type 2 diabetes mellitus
}

\author{
Yunjie Teng ${ }^{1}$, Shuxia Wang ${ }^{1}$, Ning Wang ${ }^{1}$, Muhuyati $^{2}$
}

\author{
${ }^{1}$ Center of Health Management, The First Affiliated Hospital, Xinjiang Medical \\ University, Urumqi, China \\ 2Department of No. 1 Cadre Ward Medicine, The First Affiliated Hospital, Xinjiang \\ Medical University, Urumqi, China
}

Submitted: 6 January 2018

Accepted: 4 February 2018

Arch Med Sci 2018; 14, 5: 971-978

DOI: https://doi.org/10.5114/aoms.2018.73984

Copyright $\odot 2018$ Termedia \& Banach

\section{Abstract}

Introduction: The aim of the current study was to evaluate the efficiency of the STOP-Bang questionnaire for obstructive sleep apnea (OSA) screening among Chinese patients with type 2 diabetes mellitus (T2DM).

Material and methods: A total of 262 patients with T2DM were enrolled for STOP-Bang questionnaire evaluation and polysomnography (PSG) monitoring between May 2015 and September 2016. Patients were divided into non-OSA and different OSA severity groups (mild, moderate, and severe) according to the apnea-hypopnea index (AHI). The value of the STOP-Bang questionnaire for OSA screening was evaluated.

Results: Efficiency of the STOP-Bang questionnaire for OSA screening among Chinese patients with $T 2 D M$ was as follows: when $\mathrm{AHI} \geq 5 / \mathrm{h}$, the area under the curve (AUC) was 0.825 (95\% confidence interval $(\mathrm{Cl}): 0.763-0.887, p<0.05)$, sensitivity was $85.6 \%(95 \% \mathrm{Cl}: 85.55-85.65 \%, p<0.05)$ and specificity was $60 \%$ (95\% Cl: $59.85-60.15 \%, p<0.05)$; when AHI > 15/h, the AUC was 0.856 $(95 \% \mathrm{Cl}: 0.799-0.913, p<0.05)$, sensitivity was $88.6 \%(95 \% \mathrm{Cl}: 88.55-$ $88.65 \%, p<0.05)$ and specificity was $38.4 \%(95 \% \mathrm{Cl}: 38.30-38.49 \%$, $p<0.05)$; when $\mathrm{AHI}>30 / \mathrm{h}$, the AUC was $0.891(95 \% \mathrm{Cl}: 0.836-0.946$, $p<0.05)$, sensitivity was $90.5 \%(95 \% \mathrm{Cl}: 90.44-90.56 \%, p<0.05)$, and specificity was $27 \%(95 \% \mathrm{Cl}: 26.94-27.07 \%, p<0.05)$.

Conclusions: The STOP-Bang questionnaire is an effective tool for OSA screening among Chinese patients with T2DM. A cut-off score of 3 distinguishes OSA from non-OSA with high sensitivity.

Key words: STOP-Bang questionnaire, obstructive sleep apnea, type 2 diabetes mellitus.

\section{Introduction}

Obstructive sleep apnea (OSA), characterized by apnea and hypopnea due to repeated partial or complete upper airway obstruction during sleep, is the most common chronic sleep-related breathing disorder [1, 2]. Numerous studies have revealed that OSA is independently associated with hypertension [3, 4], coronary heart diseases [5-8], arrhythmias [9], diabetes mellitus [10-13], metabolic syndrome [12, 13], stroke [14], and chronic kidney disease [15-17]. Only $4 \%$ of middle-aged men and $2 \%$ of middle-aged women with OSA have clinical symptoms [18]. The awareness

\author{
Corresponding authors: \\ Ning Wang \\ The First \\ Affiliated Hospital \\ Xinjiang Medical \\ University \\ 137 Liyushan South Road \\ 830011 Urumqi, China \\ E-mail: 13999994126@163.com \\ Muhuyati \\ E-mail: muhuyati@163.com
}


of OSA is gradually increasing; however, the rate of missed diagnosis is still high. For example, West et al. reported that about $23 \%$ of patients with type 2 diabetes mellitus (T2DM) had OSA and most of these patients were not diagnosed [19].

Numerous studies have reported that OSA is a major risk factor for insulin resistance. Indeed, OSA severity is positively correlated with the degree of insulin resistance [10]. A prior study also reported that the prevalence of OSA in Chinese T2DM patients was $17.5 \%$, which was significantly higher than the general population [20]. Furthermore, continuous positive airway pressure (CPAP) treatment, an effective therapeutic method for OSA, was beneficial for insulin sensitivity improvement, blood glucose control and hemoglobin $A_{1 c}\left(H b A_{1 c}\right)$ reduction [21-24]. Therefore, it is clinically relevant to screen OSA in diabetic patients.

Timely diagnosis and treatment of OSA among patients with T2DM is an integral part of diabetes management. However, the gold standard, in terms of overnight polysomnography (PSG), for OSA diagnosis is complex, expensive and time-consuming. Moreover, it is impossible to conduct PSG for every diabetic patient, especially those with critically ill diseases. Thus, it is necessary to design a sensitive and accurate tool for OSA screening in diabetic patients.

Various predictive models and scales have been designed for screening OSA. However, these models have not been validated in diabetic patients. For example, Zou et al. used the Epworth Sleepiness Scale (ESS) to screen OSA among general subjects with suspected OSA, but the prevalence of diabetes in the study was not reported [25]. Karakoc et al. applied the Berlin questionnaire to screen OSA in subjects who were referred to the Department of Otolaryngology, and the prevalence of diabetes was also not addressed [26]. The STOP-Bang questionnaire is a simple, feasible and efficient questionnaire for OSA screening [27], which is also increasingly applicable for OSA screening among patients with myocardial infarction [28] and obesity [29]. Prior studies also showed that the STOP-Bang questionnaire was a more accurate tool for detecting mild, moderate, and severe OSA [30]. Therefore, in the current study, we used a prospective design to evaluate the value of the STOP-Bang questionnaire for OSA screening among Chinese patients with T2DM.

\section{Material and methods}

\section{Study patients}

In-patients with T2DM were recruited at the First Affiliated Hospital of Xinjiang Medical University. From May 2015 to September 2016, all in-patients were screened for eligibility and approached for overnight PSG. The inclusion criteria were as follows: age $\geq 18$ years old, and having a documented diagnosis of T2DM in terms of treatment with anti-diabetic medications or insulin. All participants gave their informed consent before being included in the study. Patients who were previously diagnosed with OSA or other sleep-related breathing disorders, currently under CPAP treatment, unable to complete overnight PSG, or pregnant, were excluded. The study was approved by the institutional ethics review board of the First Affiliated Hospital of Xinjiang Medical University.

\section{Demographic and biochemical measurements}

Before PSG monitoring, histories of T2DM, hypertension, respiratory system diseases, and nervous system diseases as well as relevant treatment were recorded. Blood pressure, height, weight, and neck circumference were measured by trained investigators, and body mass index (BMI) was calculated using weight in kilograms divided by height in meters squared. In detail, blood pressure was measured when patients had been sitting quietly for 10 min before measurement. A suitable cuff which covered at least $80 \%$ of the circumference was placed on the non-dominant arm and two measurements with a 1-minute interval were obtained and averaged. Neck girth was measured above the thyroid cartilage with participants keeping the head up and looking straight forward, and waist circumference was measured at the midpoint between the lower margin of the last palpable rib and the top of the iliac crest.

Patients fasted for $12 \mathrm{~h}$ before blood sampling and all samples were drawn between 7 am to $8 \mathrm{am}$. Plasma glucose was measured by the glucose oxidase method using a Roche c8000 autoanalyzer. $\mathrm{HbA}_{1 c}$ was measured in whole blood using ion-exchange high-performance liquid chromatography with the Bio-Rad D10 Variant Hemoglobin Testing System.

\section{STOP-Bang questionnaire}

The Chinese STOP-Bang scale was translated by Yu et al. [31] and authorized by Frances Chung, the author of the original STOP-Bang questionnaire. All eligible patients were asked to complete the STOP-Bang questionnaire independently and underwent in-hospital overnight PSG monitoring. Affirmative answers ('Yes') on items were marked as 1 and negatives ('No') as 0 based on the scoring method for the original English scale.

\section{Polysomnography}

A portable PSG (Embletta X100) with approved liability [32] was applied. The recording configu- 
ration included two-lead electroencephalograph (EEG), two-lead electro-oculogram (EOG), jaw muscle electromyography (EMG), electrocardiogram (EKG), oral and nasal airflow, thoracic and abdominal breathing movements, oxygen saturation, body position, snoring and other PSG parameters. Patients were prohibited from drinking alcohol or taking sedative drugs on the night of PSG monitoring. The duration of monitoring was $\geq 7 \mathrm{~h}$. The PSG data were manually analyzed by senior PSG physicians, who were blinded to the results of the STOP-Bang questionnaire and other clinical information about these patients. The clinical diagnosis of OSA was defined as $\mathrm{AHI} \geq 5 / \mathrm{h}$ with fragmented sleep and daytime sleepiness. According to the American Academy of Sleep Medicine practice guidelines [33], the severity of OSA is determined by the $A H I$ : $A H I<5 / h$, none; $5-15 / h$, mild; $15-30 / h$, moderate; $\geq 30 / \mathrm{h}$, severe. The results of PSG were used to evaluate the validity of the STOP-Bang questionnaire.

\section{Statistical analysis}

SPSS 17.0 statistical software was used for all statistical analyses. All data were normally distributed with the exception of $\mathrm{AHI}$ and lowest blood oxygen saturation $\left(\mathrm{LSaO}_{2}\right)$. Normally distributed continuous variables, which were tested by the Kolmogorov-Smirnov test, were expressed as mean $\pm S D$, while continuous data with skewed distributions were expressed as median and interquartile range. Categorical variables were expressed as number and percentage of cases. Paired sample means were compared by the independent sample $t$ test or Mann-Whitney $U$ test. Rates were compared by the $\chi^{2}$ test or correction for continuity $\chi^{2}$ test. Four-fold tables were constructed according to STOP-Bang questionnaire results and PSG results, and sensitivity, specificity, positive predictive value (PPV) and negative predictive value (NPV) were calculated using receiver operating characteristic (ROC) curve analysis of area under the curve (AUC). A logistic regression analysis model of ordinal categorical data was constructed to assess the corresponding probabilities of different OSA degrees (no, mild, moderate, or severe) for specific STOP-Bang scores. A two-sided $p$-value $<0.05$ was considered as statistically significant.

\section{Results}

\section{General characteristics}

A total of 739 diabetic patients (112 were newly diagnosed) were approached for participation in this study, 185 were excluded and 156 declined to participate. Seventeen patients did not complete the STOP-Bang questionnaire and
113 did not complete PSG monitoring. In total, 268 patients completed the questionnaire and portable PSG, among whom 5 were excluded because the overall sleep time was too short (<240 min). After analysis of PSG data, 1 patient was found to have central sleep apnea and was excluded (Figure 1).

The remaining 262 patients were included in the final analysis. Among them, 191 (72.9\%) were male and $71(27.1 \%)$ were female, with the average age of $49.2 \pm 10.6$ years. The mean BMI was $28.3 \pm 3.88 \mathrm{~kg} / \mathrm{m}^{2}$, the mean fasting blood glucose was $6.03 \pm 1.95 \mathrm{mmol} / \mathrm{l}$, and the average duration of T2DM was 8.5 years. Of the 262 patients, 40 patients did not have OSA and 222 had OSA; 55 had mild, 83 moderate and 84 severe OSA.

\section{Comparisons between non-OSA and OSA patients}

As shown in Table I, compared to non-OSA patients, those with OSA were older, more likely to be male, and had significantly higher BMI, neck girth and waist circumference ( $p<0.05$ for all comparisons). In addition, patients with OSA had higher systolic and diastolic blood pressure and fasting blood glucose, while no significant difference in $\mathrm{HbA}_{1 \mathrm{c}}$ was observed. As expected, OSA patients had significantly higher median AHI (24.5/h

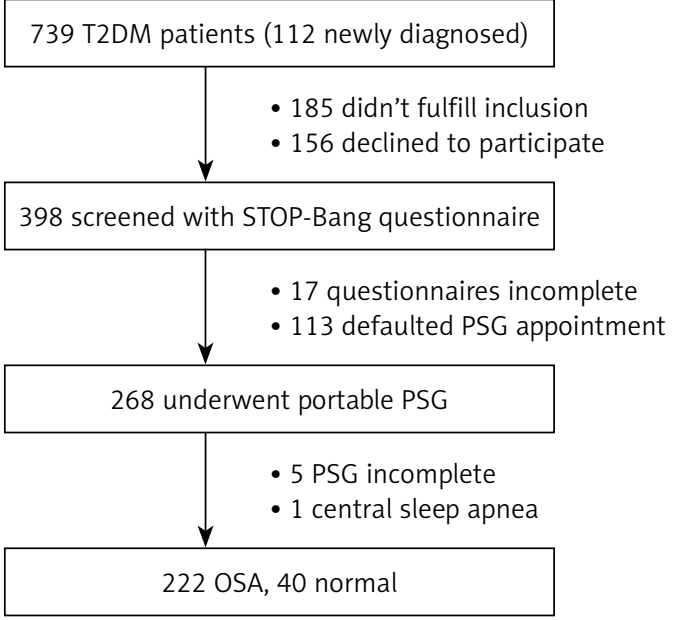

Figure 1. Study flow chart. A total of 739 patients were approached for participation in this study, of whom 112 were newly diagnosed with T2DM, 185 were excluded, and 156 declined to participate. Seventeen patients did not complete the questionnaire and 113 did not complete PSG. In total, 268 patients completed the questionnaire and portable PSG, among whom 5 were excluded because the overall sleep time was too short. After the manual analysis of PSG data, 1 patient was found to have central sleep apnea and excluded. The remaining 262 patients were included in the analysis. Of the 262 patients, 40 did not have OSA and 222 patients had OSA

T2DM - type 2 diabetes mellitus, PSG - polysomnography, OSA - obstructive sleep apnea. 
Yunjie Teng, Shuxia Wang, Ning Wang, Muhuyati

Table I. Comparisons between non-OSA and OSA patients

\begin{tabular}{|c|c|c|c|c|}
\hline Variables & Non-OSA $(n=40)$ & OSA $(n=222)$ & $t / \chi^{2} / Z$ & $P$-value \\
\hline Age [years] & $42.53 \pm 11.80$ & $50.39 \pm 9.97$ & -4.46 & $<0.001$ \\
\hline Gender (F/M) & $25 / 15$ & $46 / 176$ & 29.95 & $<0.001$ \\
\hline BMI $\left[\mathrm{kg} / \mathrm{m}^{2}\right]$ & $27.25 \pm 3.54$ & $28.63 \pm 3.97$ & -2.06 & 0.04 \\
\hline $\mathrm{BMI}>28 \mathrm{~kg} / \mathrm{m}^{2}, n(\%)$ & $14(35)$ & $106(47.7)$ & 2.13 & 0.136 \\
\hline $\mathrm{BMI}>30 \mathrm{~kg} / \mathrm{m}^{2}, n(\%)$ & $9(22.5)$ & $69(31.1)$ & 1.19 & 0.275 \\
\hline $\mathrm{BMI}>35 \mathrm{~kg} / \mathrm{m}^{2}, n(\%)$ & $1(2.5)$ & $14(6.3)$ & 0.34 & 0.559 \\
\hline Neck circumference $[\mathrm{cm}]$ & $37.28 \pm 2.85$ & $39.16 \pm 2.77$ & -3.93 & $<0.001$ \\
\hline Waist circumference $[\mathrm{cm}]$ & $97.15 \pm 10.12$ & $101.6 \pm 9.69$ & -2.66 & 0.008 \\
\hline Mean SBP [mm Hg] & $129.35 \pm 15.71$ & $146.81 \pm 16.24$ & -6.29 & $<0.001$ \\
\hline Mean DBP [mm Hg] & $76.56 \pm 7.12$ & $92.15 \pm 10.36$ & -9.13 & $<0.001$ \\
\hline Fasting glucose [mmol/l] & $5.52 \pm 2.06$ & $6.48 \pm 2.06$ & -2.71 & 0.01 \\
\hline $\mathrm{HbA}_{1 \mathrm{c}}(\%)$ & $8.06 \pm 1.7$ & $8.3 \pm 1.52$ & -0.50 & 0.368 \\
\hline AHI median $\left(25-75^{\text {th }}\right)$ & $4.45(3.55-4.70)$ & $24.5(15.3-36.9)$ & -7.13 & $<0.001$ \\
\hline $\mathrm{LSpO}_{2}(\%)$ & $86.5(86.88)$ & $81.0(76.75)$ & 3.26 & $<0.001$ \\
\hline STOP score & $1.50 \pm 0.93$ & $2.33 \pm 1.06$ & -4.64 & $<0.001$ \\
\hline STOP-Bang score & $2.23 \pm 1.00$ & $3.91 \pm 1.41$ & -7.21 & $<0.001$ \\
\hline
\end{tabular}

OSA - obstructive sleep apnea, F/M - female/male, BMI - body mass index, SBP-systolic blood pressure, DBP - diastolic blood pressure, $H b A_{1 c}$ - hemoglobin $A_{1 c}, A H I$ - apnea hypopnea index, $L S p O_{2}$ - lowest saturated arterial oxygen pressure $\left(\mathrm{pO}_{2}\right)$.

vs. $4.45 / \mathrm{h}$ ) and lower saturated arterial oxygen pressure $\left(\mathrm{LSpO}_{2}\right)$ ( $81 \%$ vs. $\left.86.5 \%\right)$. The STOP score $(2.33 \pm 1.06$ vs. $1.50 \pm 0.93)$ and STOP-Bang score (3.91 \pm 1.41 vs. $2.23 \pm 1.00$ ) were also significantly higher in OSA patients versus non-OSA patients ( $p<0.001$ for all comparisons).

Table II. Questionnaire characteristics

\begin{tabular}{|lc|}
\hline Questionnaire & $\begin{array}{c}\text { No. (\%) of patients } \\
\text { answering yes }\end{array}$ \\
\hline S: Snore loudly? & $180(68.7)$ \\
\hline $\begin{array}{l}\text { T: Tired or sleepy during } \\
\text { daytime? }\end{array}$ & $113(43.1)$ \\
\hline O: Observed apnea? & $107(40.8)$ \\
\hline P: High blood pressure? & $179(68.3)$ \\
\hline B: BMI > 35 kg/m²? & $105(40.1)$ \\
\hline A: Age > 50 years? & $67(25.6)$ \\
\hline N: Neck circumference $>40 \mathrm{~cm} ?$ & $191(72.9)$ \\
\hline $\begin{array}{l}\text { G: Gender male? } \\
\text { STOP-Bang score }\end{array}$ & $3.65 \pm 1.48$ \\
\hline No. of patients classified as high risk: \\
\hline \begin{tabular}{l} 
STOP-Bang \\
\hline
\end{tabular}
\end{tabular}

$B M I-$ body mass index.

\section{Score distribution of the STOP-Bang scale}

Of the 262 diabetic patients screened for OSA by the STOP-Bang questionnaire, 206 (78.6\%) were classified as at high risk of OSA as defined by the STOP-Bang score $\geq 3$. The positive rate for each individual item is shown in Table II and the distribution of STOP-Bang questionnaire scores is presented in Figure 2.

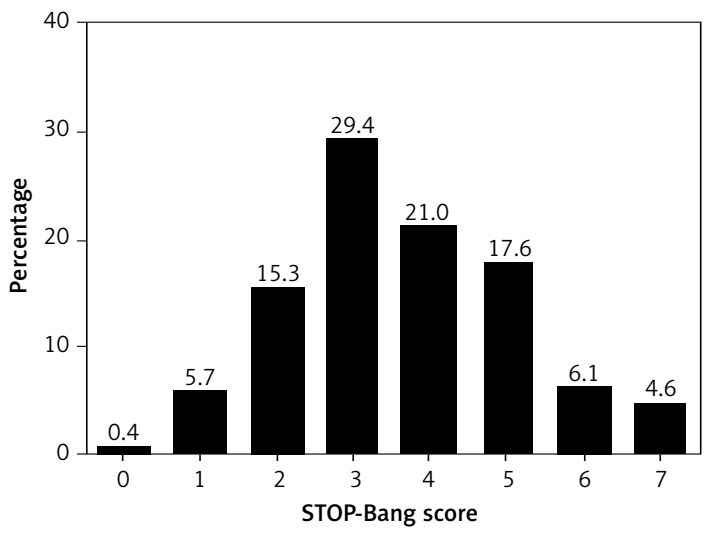

Figure 2. Distribution of STOP-Bang score in 262 T2DM patients. There was 1 person with a score of 0,15 people with a score of 1,40 people with a score of 2, 77 people with a score of 3, 55 people with a score of 4,46 people with a score of 5,16 people with a score of 6 , and 12 people with a score of $7.206(78.6 \%)$ were at high risk of OSA as defined by scores $\geq 3$ 
Table III. Diagnostic parameters of STOP-Bang scale

\begin{tabular}{|cccccc|}
\hline AHI & Score & Sensitivity & Specificity & PPV & NPV \\
\hline$\geq 5$ & $\geq 3$ & $85.6(85.55-85.65)$ & $60(59.85-60.15)$ & $92.2(92.16-92.24)$ & $42.9(42.77-43.03)$ \\
\cline { 2 - 6 } & $\geq 4$ & $56.3(56.24-56.37)$ & $90(89.91-90.09)$ & $96.9(96.87-96.93)$ & $27.1(27.02-27.18)$ \\
\cline { 2 - 6 } & $\geq 5$ & $33.3(33.24-33.36)$ & $100(100-100)$ & $100(100-100)$ & $21.3(21.24-21.36)$ \\
\cline { 2 - 6 }$>15$ & $\geq 6$ & $12.6(12.56-12.64)$ & $100(100-100)$ & $100(100-100)$ & $17.1(17.05-17.15)$ \\
\cline { 2 - 6 } & $\geq 3$ & $88.6(88.55-88.65)$ & $38.4(38.30-38.49)$ & $71.8(71.74-71.86)$ & $66.1(65.98-66.22)$ \\
\cline { 2 - 6 } & $\geq 5$ & $38.3(38.23-38.37)$ & $89.5(89.44-89.56)$ & $86.5(86.42-86.58)$ & $45.2(45.13-45.27)$ \\
\hline$>30$ & $\geq 3$ & $90.5(90.44-90.56)$ & $27(26.94-27.07)$ & $36.9(36.83-36.97)$ & $85.7(85.61-85.79)$ \\
& $\geq 4$ & $76.2(76.11-76.29)$ & $63.5(63.43-63.57)$ & $49.6(49.51-49.69)$ & $85(84.94-85.06)$ \\
& $\geq 5$ & $52.4(52.29-52.51)$ & $83.1(83.05-83.16)$ & $59.5(59.39-59.61)$ & $78.7(78.64-78.76)$ \\
\cline { 2 - 6 } & $\geq 6$ & $21.4(21.31-21.49)$ & $94.4(94.37-94.43)$ & $64.3(64.12-64.48)$ & $71.8(71.74-71.86)$ \\
\hline
\end{tabular}

AHI - apnea-hypopnea index, PPV - positive predictive value, NPV - negative predictive value.

Value of STOP-Bang scale for OSA screening

Receiver operator curve analysis of the STOPBang score was conducted to establish the cutoff for OSA screening in patients with T2DM. As presented in Table III, the AUC was $0.825(0.763$, $0.887)$ for $\mathrm{AHI} \geq 5 / \mathrm{h}, 0.856(0.799,0.913)$ for $\mathrm{AHI}$ $>15 / \mathrm{h}$, and $0.891(0.836,0.946)$ for $\mathrm{AHI}>30 / \mathrm{h}$. The individual sensitivity, specificity, PPV, and NPV of the STOP-Bang score for OSA screening within each category of OSA are presented in Table III.

The OSA severity distribution according to the STOP-Bang score is presented in Table IV. The predicted probability of OSA is presented in Table $\mathrm{V}$ and Figure 3. Overall, with increase of the STOP-Bang score, the probability of having no OSA decreased from $66.59 \%$ to $0.49 \%$ and the probability of having severe OSA increased from $2.45 \%$ to $91.08 \%$. When the total score was $1-2$, the probability of having mild OSA was $30.5-33.19 \%$, and the probability of having mild OSA gradually decreased with increase of the total STOP-Bang score. When the total STOP-Bang score was 3-4, the probability of having moderate OSA was $35.17-38.09 \%$ and the probability gradually decreased as the total STOP-Bang score increased.

\section{Discussion}

Our current study shows that the sensitivity of the STOP-Bang questionnaire for OSA screening in diabetic patients was $85.6-90.5 \%$, which was consistent with a previous screening study in the

Table IV. OSA severity distribution according to STOP-Bang questionnaire score

\begin{tabular}{|lcccc|}
\hline STOP-Bang score & No OSA & Mild & Moderate & Severe \\
\hline 0 & 1 & 0 & 0 & 0 \\
\hline 1 & 9 & 3 & 1 & 2 \\
\hline 2 & 14 & 10 & 28 & 6 \\
\hline 3 & 12 & 25 & 24 & 20 \\
\hline 4 & 4 & 7 & 13 & 26 \\
\hline 5 & 0 & 7 & 4 & 10 \\
\hline 6 & 0 & 2 & 3 & 8 \\
\hline 7 & 0 & 1 & 0 & 0 \\
\hline 8 & 0 & 0 & 83 & 84 \\
\hline Total & 40 & 55 & & 12 \\
\hline OSA & & & 25 & 26 \\
\hline
\end{tabular}

OSA - obstructive sleep apnea. 
Table V. Probability of OSA severity distribution according to STOP-Bang questionnaire score (\%)

\begin{tabular}{|lcccc|}
\hline STOP-Bang score & No OSA (\%) & Mild (\%) & Moderate (\%) & Severe (\%) \\
\hline 0 & 66.59 & 22.25 & 8.71 & 2.45 \\
\hline 1 & 48.47 & 30.50 & 15.97 & 5.05 \\
\hline 2 & 30.74 & 33.19 & 25.94 & 10.13 \\
\hline 3 & 17.32 & 28.23 & 35.17 & 19.28 \\
\hline 4 & 9.00 & 19.31 & 38.09 & 33.60 \\
\hline 5 & 4.46 & 11.25 & 32.55 & 51.75 \\
\hline 6 & 2.15 & 5.93 & 22.47 & 69.45 \\
\hline 7 & 1.03 & 2.95 & 13.21 & 82.81 \\
\hline 8 & 0.49 & 1.43 & 7.00 & 91.08 \\
\hline
\end{tabular}

general Chinese population. In addition, compared to the general Chinese population [34], the AUC was also substantially higher in diabetic patients (0.825-0.891 vs. $0.815-0.751)$, strongly indicating that the STOP-Bang questionnaire is effective for OSA screening in patients with T2DM.

Obstructive sleep apnea is a well-known risk factor for cardiovascular and renal diseases. However, a substantial number of patients with OSA have no clinical symptoms and have not been diagnosed yet. With increasing age and obesity, the incidence and prevalence of OSA are increasing dramatically; therefore, it is clinically important to screen OSA so as to reduce the health and eco-

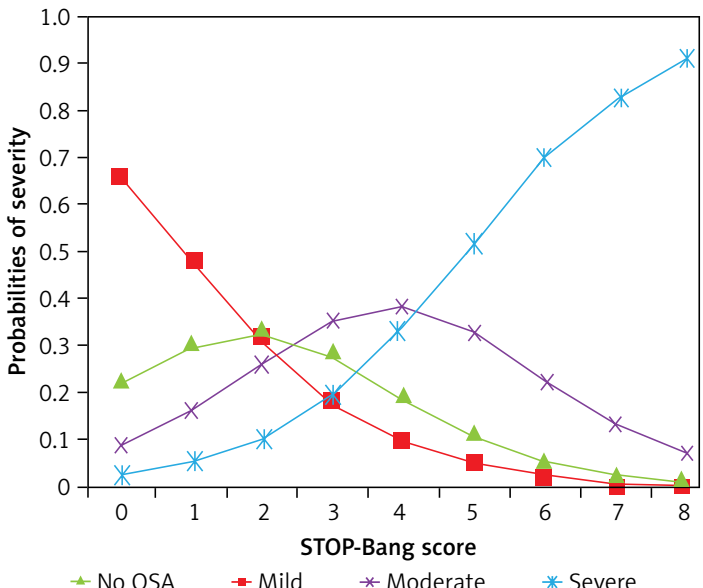

Figure 3. Probability of different severities of OSA according to STOP-Bang questionnaire score. With increasing scores, the probability of no OSA decreased from $66.59 \%$ to $0.49 \%$ and the probability of severe OSA increased from $2.45 \%$ to $91.08 \%$. When the total score was $1-2$, the probability of mild OSA was $30.5-33.19 \%$. The probability of mild OSA gradually decreased with increasing total STOP-Bang score. When the total STOP-Bang score was $3-4$, the probability of moderate OSA was $35.17-38.09 \%$, higher than the probabilities of other severities. The probability gradually decreased as the total score increased nomic burden of OSA. Polysomnography has been used for OSA diagnosis and evaluation. However, PSG monitoring is complex, expensive and time-consuming. In addition, it is not feasible for population screening due to the paucity of PSG facilities in rural areas. The STOP-Bang questionnaire has been designed and used for OSA screening in the general population with an acceptable sensitivity and accuracy.

Notably, OSA is highly prevalent in patients with diabetes, and OSA combined with diabetes imposes greater cardiovascular risk than that of each individual morbidity. Therefore, it is clinically important to screen OSA in diabetic patients. Most prior studies used PSG to screen and diagnose OSA in patients with diabetes, and whether the STOP-Bang questionnaire is also applicable for OSA screening in diabetic patients is unknown. In our current study, we evaluated the validity of the STOP-Bang questionnaire for OSA screening, which was later confirmed using PSG monitoring.

Our study showed that the probability of having OSA increased with an increasing STOP-Bang score. The sensitivity was highest using a diagnostic cut-off value of 3 (85.6\% for mild, $88.6 \%$ for moderate and $90.5 \%$ of severe), with a specificity of $60 \%, 38.4 \%$, and $27 \%$, respectively. Positive rates for male gender, loud snoring and having high blood pressure were 191 (72.9\%), 180 (68.7\%), and 179 (68.3\%) respectively, indicating that existence of these factors was justifiable to conduct OSA screening in diabetic patients using the STOP-Bang questionnaire.

It has been suggested that there were differences in prevalence of cardiovascular risk factors including OSA between the general male and female populations [35]. Consistent with prior reports [36, 37], we also observed that compared to the female participants with OSA, the percentage of male participants with OSA was also signifi- 
cantly higher, indicating that male gender might be a potential key risk factor.

Importantly, as recently reported by Westlake et al. [38], we should be cautious that a false negative result from an OSA screening questionnaire might have serious clinical and ethical implications for diabetic patients who are suffering from moderate or severe OSA but who are deprived of therapy if inappropriately classified as being at low risk. In our current study, 39 and 20 patients with moderate or severe OSA, respectively, had a STOP-Bang score of 3 or less. We found that compared to the moderate or severe OSA patients but whose STOP-bang score was $>3$, these lower-score patients had a higher $\mathrm{HbA}_{1 c}$ level, suggesting that $\mathrm{HbA}_{1 c}$ might be useful for including in the STOP-bang questionnaire for OSA screening in diabetic patients, which deserves further elucidation in a future study. Collectively, the results from our current study indicated that a BANGSTOP score $>3$ was significantly associated with a high probability of OSA. However, when the BANG-STOP is $<3$, one should bear in mind that OSA could not be completely excluded due to the possibility of a false negative.

Finally, several studies have validated the use of the STOP-BANG score. For example, Chung et al. [39] enrolled 310 obese Canadian patients and found that a STOP-Bang score of 4 had high sensitivity $(87.5 \%)$ and high negative predictive value $(90.5 \%)$ for identifying severe OSA. In another study conducted in the Korean population, Kim et al. [40] reported that the STOP-Bang questionnaire had high sensitivity ( $97 \%$ for $\mathrm{AHI} \geq 5 / \mathrm{h}$ and $98 \%$ for $\mathrm{AHI} \geq 15 / \mathrm{h}$ ) for OSA screening in those with suspected OSA. These findings combined with our current results strongly support the notion that the STOP-Bang questionnaire is a useful tool for OSA screening in different populations.

There were some limitations of the current study. First, the patients enrolled were Chinese, and the findings could not be extrapolated to other ethnic groups. Second, the proportion of T2DM patients in this study diagnosed with OSA by PSG was substantially higher than in the general T2DM population because all patients enrolled satisfied the inclusion criteria for PSG. Last but not least, most patients completing the STOP-Bang questionnaire and PSG monitoring had subjective clinical symptoms and had multiple risk factors of OSA, and therefore inclusion bias might be unavoidable.

In conclusion, the STOP-Bang questionnaire has high sensitivity for OSA screening and severity assessment among Chinese diabetic patients. In addition, the STOP-Bang questionnaire is particularly useful for male patients, and those who have hypertension and snore loudly. Screening OSA using the STOP-Bang questionnaire may be a potential important screening tool for early identification of OSA in diabetic patients.

\section{Conflict of interest}

The authors declare no conflict of interest.

\section{References}

1. Young T, Palta M, Dempsey J, Skatrud J, Weber S, Badr S. The occurrence of sleep-disordered breathing among middle-aged adults. N Engl J Med 1993; 328: 1230-5.

2. Epstein LJ, Kristo D, Strollo PJ Jr, et al. Clinical guideline for the evaluation, management and long-term care of obstructive sleep apnea in adults. J Clin Sleep Med 2009; 5: 263-76.

3. Peppard PE, Young T, Palta M, Skatrud J. Prospective study of the association between sleep disordered breathing and hypertension. N Engl J Med 2000; 342: 1378-84.

4. Chobanian AV, Bakris GL, Black HR, et al. The Seventh Report of the Joint National Committee on Prevention, Detection, Evaluation, and Treatment of High Blood Pressure: the JNC 7 report. JAMA 2003; 289: 2560-72.

5. Bounhoure JP, Galinier M, Didier A, Leophonte P. Sleep apnea syndromes and cardiovascular disease. Bull Acad Natl Med 2005; 189: 445-59.

6. Marin JM, Carrizo SJ, Vicente E, Agusti AG. Long-term cardiovascular outcomes in men with obstructive sleep apnoea-hypopnoea with or without treatment with continuous positive airway pressure: an observational study. Lancet 2005; 365: 1046-53.

7. Kohler M, Stradling JR. Mechanisms of vascular damage in obstructive sleep apnea. Nat Rev Cardiol 2010; 7: 677-85.

8. Porto F, Sakamoto YS, Salles C. Association between obstructive sleep apnea and myocardial infarction: a systematic review. Arq Bras Cardiol 2017; 108: 361-9.

9. Rossi VA, Stradling JR, Kohler M. Effects of obstructive sleep apnoea on heart rhythm. Eur Respir J 2013; 41: 1439-51.

10. Ip MS, Lam B, Ng MM, Lam WK, Tsang KW, Lam KS. Obstructive sleep apnea is independently associated with insulin resistance. Am J Repir Crit Care Med 2002; 165: 670-6.

11. Mazaherioun M, Saedisomeolia A, Javanbakht MM, et al. Beneficial effects of $n-3$ polyunsaturated fatty acids on adiponectin levels and AdipoR gene expression in patients with type 2 diabetes mellitus: a randomized, placebo-controlled, double-blind clinical trial. Arch Med Sci 2017; 13: 716-24.

12. Katsiki N, Athyros VG, Karagiannis A, Mikhailidis DP. Characteristics other than the diagnostic criteria associated with metabolic syndrome: an overview. Curr Vasc Pharmacol 2014; 12: 627-41.

13. Qian Y, Xu H, Wang Y, Yi H, Guan J, Yin S. Obstructive sleep apnea predicts risk of metabolic syndrome independently of obesity: a meta-analysis. Arch Med Sci 2016; 12: 1077-87.

14. Barbarash O, Gruzdeva O, Uchasova E, et al. Biochemical markers of type 2 diabetes as a late complication of myocardial infarction: a case-control study. Arch Med Sci 2017; 13: 311-20.

15. Chou YT, Lee PH, Yang CT, et al. Obstructive sleep apnoea: a stand-alone risk factor for chronic kidney disease. Nephrol Dial Transplant 2001; 26: 2244-50.

16. Iseki K, Tohyama K, Matsumoto T, Nakamura H. High prevalence of chronic kidney disease among patients 
with sleep related breathing disorder (SRBD). Hypertens Res 2008; 31: 249-55.

17. Faulx MD, Storfer-Isser A, Kirchner HL, Jenny NS, Tracy RP, Redline S. Obstructive sleep apnea is associated with increased urinary albumin excretion. Sleep 2007; 30: 923-9.

18. Young T, Palta M, Dempsey J, Skatrud J, Weber S, Badr S. The occurrence of sleep-disordered breathing among middle-aged adults. N Engl J Med 1993; 328: 1230-5.

19. West SD, Nicoll DJ, Stradling JR. Prevalence of obstructive sleep apnoea in men with type 2 diabetes. Thorax 2006; 61: 945-50.

20. Stevens RJ, Coleman RL, Adler Al, et al. Risk factors for myocardial infarction case fatality and stroke case fatality in type 2 diabetes. Diabetes Care 2004; 27: 201-7.

21. Harsch IA, Schahin SP, Brückner K, et al. The effect of continuous positive airway pressure treatment on insulin sensitivity in patients with obstructive sleep apnoea syndrome and type 2 diabetes. Respiration 2004; 71: 252-9.

22. Babu AR, Herdegen J, Fogelfeld L, Shott S, Mazzone T. Type 2 diabetes, glycemic control, and continuous positive airway pressure in obstructive sleep apnea. Arch Intern Med 2005; 165: 447-52.

23. Hassaballa HA, Tulaimat A, Herdegen JJ, Mokhlesi B. The effect of continuous positive airway pressure on glucose control in diabetic patients with severe obstructive sleep apnea. Sleep Breath 2005; 9: 176-80.

24. Malik JA, Masoodi SR, Shoib S. Obstructive sleep apnea in type 2 diabetes and impact of continuous positive airway pressure therapy on glycemic control. Indian J Endocrinol Metab 2017; 21: 106-12.

25. Zou J, Guan J, Yi H, et al. An effective model for screening obstructive sleep apnea: a large-scale diagnostic study. PLoS One 2013; 8: e80704.

26. Karakoc O, Akcam T, Genc H, Yetkin S, Piskin B, Gerek M Use of the Berlin Questionnaire to screen at-risk patients for obstructive sleep apnea. B-ENT 2014; 10: 21-5.

27. Nagappa M, Liao P, Wong J, et al. Validation of the STOPBang questionnaire as a screening tool for obstructive sleep apnea among different populations: a systematic review and meta-analysis. PLoS One 2015; 10 e0143697.

28. McCormack DJ, Pabla R, Babu MH, et al. Undiagnosed sleep apnoea syndrome in patients with acute myocardial infarction: potential importance of the STOP-BANG screening tool for clinical practice. Int J Cardiol 2012; 155: 342-3.

29. Chung F, Yang Y, Liao P. Predictive performance of the STOP-Bang score for identifying obstructive sleep apnea in obese patients. Obes Surg 2013; 23: 2050-7.

30. Chiu HY, Chen PY, Chuang LP, et al. Diagnostic accuracy of the Berlin questionnaire, STOP-BANG, STOP, and Epworth sleepiness scale in detecting obstructive sleep apnea: a bivariate meta-analysis. Sleep Med Rev 2017; 36: 57-70.

31. Yu Y, Mei W, Cui Y. Primary evaluation of the simplified Chinese version of STOP-Bang scoring model in predicting obstructive sleep apnea hypopnea syndrome. Lin Chung Er Bi Yan Hou Tou Jing Wai Ke Za Zhi 2012; 26: 256-9.

32. Chung F, Liao P, Sun Y, et al. Perioperative practical experiences in using a level 2 portable polysomnography Sleep Breath 2011; 15: 367-75.

33. Sleep-related breathing disorders in adults: recommendations for syndrome definition and measurement techniques in clinical research. The Report of an Ameri- can Academy of Sleep Medicine Task Force [No authors listed]. Sleep 1999; 22: 667-89.

34. Luo J, Huang R, Zhong X, Xiao Y, Zhou J. Value of STOPBang questionnaire in screening patients with obstructive sleep apnea hypopnea syndrome in sleep disordered breathing clinic. Chin Med J (Engl) 2014; 127 1843-8.

35. Katsiki N, Mikhailidis DP. Emerging vascular risk factors in women: any differences from men? Curr Med Chem 2015; 22: 3565-79.

36. Theorell-Haglöw J, Miller CB, Bartlett DJ, Yee BJ, Openshaw HD, Grunstein RR. Gender differences in obstructive sleep apnoea, insomnia and restless legs syndrome in adults - What do we know? A clinical update. Sleep Med Rev 2018; 38: 28-38.

37. Wimms A, Woehrle H, Ketheeswaran S, Ramanan D, Armitstead J. Obstructive sleep apnea in women: specific issues and interventions. Biomed Res Int 2016; 2016 1764837.

38. Westlake K, Polak J. Screening for obstructive sleep apnea in type 2 diabetes patients - questionnaires are not good enough. Front Endocrinol (Lausanne) 2016; 7: 124

39. Chung F, Yang Y, Liao P. Predictive performance of the STOP-Bang score for identifying obstructive sleep apnea in obese patients. Obes Surg 2013; 23: 2050-7.

40. Kim B, Lee EM, Chung YS, Kim WS, Lee SA. The utility of three screening questionnaires for obstructive sleep apnea in a sleep clinic setting. Yonsei Med J 2015; 56 : 684-90. 